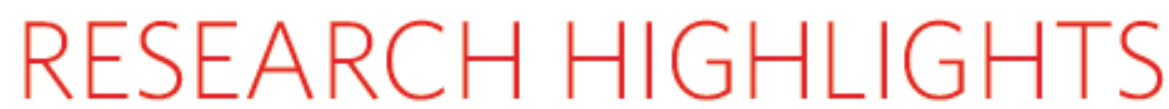

\title{
Skeleton clocks
}

Cell 122, 803-815(2005)

The genes that make the body's circadian

clock tick are also involved in controlling bone

formation, according to a team led by Gerard

Karsenty at Baylor College of Medicine in

Houston, Texas. The findings offer avenues for

tackling bone diseases such as osteoporosis in

which bone density (pictured) decreases.

Boneformation has a daily rhythm, so

Karsenty's team studied mice that had been

genetically engineered to lack key clock genes.

They found that such mice had heavier bones

because they had more bone-building cells.

The clock-gene proteins respond to signals

from the hormone leptin and control the

proliferation of thesecells. Intriguingly, bone

seems to be the only tissue where leptin acts

through this pathway, suggesting this is an

evolutionarily ancient and impor tant aspect

of leptin biology.

\section{CHEMICAL BIOLOGY}

\section{Genomic miners}

Nature Chem. Bial dot.10.1038/nchembio731 (2005)

Finding natural compounds that have important medicinal properties has always been a laborious process of searching and purification.

Now, chemists have a powerful tool to speed up their hunts. A team at the University of Warwick, UK, has used the genome sequence of a bacterium to predict that it makes a particular molecule.

The team, led by Gregory Challis, studied DNA sequences from Streptonyces coelicolor (pictured below) and identified clusters of genes involved in biosynthesis. Using these, the group predicted the existence of an unknown molecule, which it then isolated from the bacteria and named coelichelin. This technique, which has been dubbed

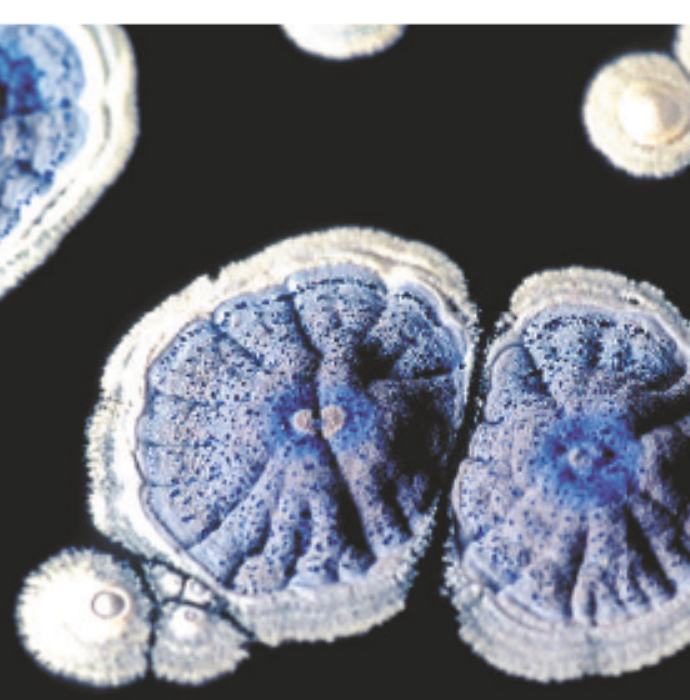

'genome mining', could be used to discover other natural products.

\section{GENETICS}

\section{BRCA2 risk for men}

J. Med. Gen. 42, 711-719(2005)

The BRCA2 gene, which has been linked to hereditary breast cancer in women, is associated with an increased risk of prostate and pancreatic cancers in men, says a

Dutch study.

Flora van Leeuwen of the Netherlands Cancer Institute in Amsterdam and her colleagues investigated 139 families with 66 different mutations of the BRCA2 gene. They studied the incidence of cancers among family members who had a $50 \%$ chance of carrying the mutant gene. The team compared these incidences with those expected in the general population and found increased risks of pancreatic and prostate cancers in men under the age of 65 . Male BRCA2 carriers may also have a greater risk of bone and throat cancers.

\section{CELL BIOLOGY}

\section{Bound and gagged}

\section{J. Cell Bial 170, 733-744 (2005)}

Cells can control the activity of enzymes by locking them up in a nuclear structure called the nucleolus, say researchers in Canada.

Stephen Lee and colleagues from the University of Ottawa, Ontario, studied two enzymes called ubiquitin ligases. When active, these attach a molecule called ubiquitin to proteins. This alters a protein's destiny, often signalling its destruction.
The enzymes studied are normally mobile and control the addition of ubiquitin to HIF (hypoxia-inducible factor) and p53, a tumour-suppressor protein. But when temporarily captured in the nucleolus, the enzymes are denied access to these molecules. The researchers propose that cells have evolved a mechanism to regulate the function of proteins by switching the enzymes between mobile and static states.

\section{NEUROBIOLOGY}

\section{Wrap artist}

Neuron 47,681-694 (2005)

A gene known as neuregulin helps to provide the insulating coat that certain nerve cells require. James Salzer of the New York University School of Medicine and his co-workers show that switching on this gene triggers the production of myelin. This fatty material envelopes some neurons and allows them to send signals up to 100 times faster than their bare counterparts. The team studied mouse neurons that are normally myelinated but had been engineered to lack both copies of the neuregulin-1 type III gene; these cells remained bare. The researchers also discovered that neurons that are usually unmyelinated acquire the protective layer when altered to express the gene.

\section{STEM CELLS}

\section{Keep your options open}

Cell 122, 1-10 (2005)

One big question about embryonic stem cells is how they maintain their potential to form 
any cell type in the body - an ability known as pluripotency. Now, a team headed by Richard Young at the Massachusetts Institute of Technology sheds light on the cellular control circuits involved.

The researchers studied three proteins called OCT4, SOX2 and NANOG, which control gene activity and are known to be important for pluripotency. Working with human stem cells, the team scanned the whole genome and identified the genes controlled by these proteins.

In many cases, the three proteins acted together on the same genes, activating those involved in pluripotency and division, and repressing those that direct embryonic development.

\section{IMMUNOLOGY}

\section{Barrier method}

\section{Nature Immunol 6,995-1001 (2005)}

A newly discovered 'firewall' that protects our cells against viral invasion could be exploited to develop antiviral drugs, say US researchers.

Roughly half of the viruses that cause human disease are surrounded by a membrane. These invaders, which include flu viruses and HIV, need to fuse their membranes with those of their host cells to enter them.

A team led by Leonid Chernomordik at the National Institutes of Health in Bethesda, Maryland, has found that a set of peptides called defensins, which form part of our innate immune system, can block this fusion. In tests on cultured human cells, they showed that defensins reversibly link cell surface proteins together, forming a barrier that stops the membranes getting close enough to fuse.

\section{CANCER}

\section{A sticky situation}

Nature Genet.doi:10.1038/ng1635 (2005) A variant of a gene called Sipa 1 seems to play a key role in helping tumours to spread. Kent Hunter of the National Cancer Institute in Bethesda, Maryland, and his colleagues have found that damping the expression of Sipa1 significantly slows tumour spread in mice. And engineering tumour cells to make extra Sipa1 protein doubles the chance that they grow and disperse. The team shows this extra protein can cause a cell to lose its adhesive properties, perhaps freeing it to colonize other organs. Tumours that have spread from human prostate cancers also express abnormally high levels of Sipa1.

\section{PLANETARY SCIENCE}

\section{Dust buster}

Science doi:10.1126/science.1118923 (2005)

On 4 July 2005, NASA's Deep Impact mission slammed a probe into Tempel 1 to find out what lies in a comet's interior. The resulting series of papers reveal detailed quantitative data on the comet's composition and structure. In an overview paper, Michael A'Hearn of the University of Maryland, College Park, and his colleagues describe how Tempel 1 is covered in impact craters, which have never been seen before on comets. The surface of Tempel 1 has surprising features, with both old- and young-looking terrains, and signs of past geological processes. The comet consists of very fine, loose particles, and its interior contains organic compounds.

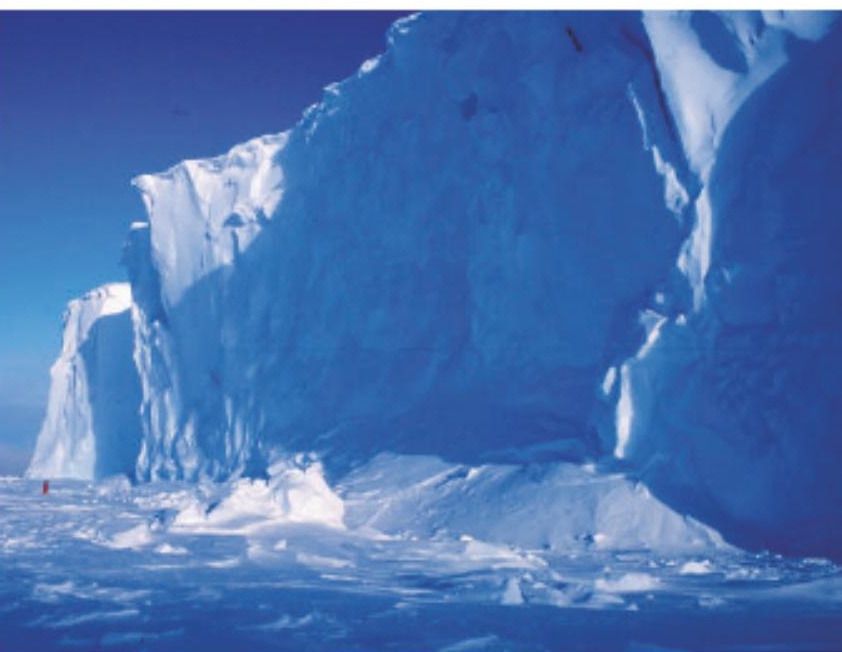

GEOMORPHOLOGY

\section{Chillout dunes}

Gealogy 33, 753-756 (2005)

The last place most people would look for an ice sheet, such as the one pictured above, is the Sahara Desert. But that is where Julien Moreau of the School and Observatory of Earth Sciences in Strasbourg and his colleagues have found one. It isn't there any more, naturally; it existed about 440 million years ago, when Africa was part of the supercontinent of Gondwana situated over the South Pole.

Moreau and his team have found signatures of glaciation - scratches and ridges in rocks and elongated deposits called drumlins - near the town of Ghat on the border of Algeria and Libya.

These features were made by an ice stream, a fast-flowing section of an ice sheet, and they are the oldest clear evidence of such streams yet found.

\section{JOURNAL CLUB}

\section{Frank Wilczek \\ Massachusetts Institute of Technology, Cambridge, USA}

The promise that anyon particles hold for quantum computing excites the physicist who named them.

When I first worked on - and named - anyons in the early 1980 s, I thought these particles, whose quantum behaviour goes beyond the familiar categories of bosons and fermions, were an interesting theoretical curiosity.

To my amazement, anyons soon appearedinthe theory of the quantum Hall effect, which describes the odd modifications of electronics that occur at low temperatures instrongmagnetic fields. Butrelevant experiments are difficult, andhave beena long time coming.

Now Fernando Camino of Stony Brook University in New York and colleagues report observations of peculiarities in the quantum Hall effect that they interpretas the first experimental indication of anyon behaviour (http://wrww.arxiv.org/ cond-mat/0504341; 2005). In a separate paper (http://wwwwarxiv. org/cond-mat/0412343; 2005), Sankar Das Sarma from the University of Maryland and his colleaguespropose a refined experimental arrangement, which could be used both to clarify this behaviour and tomake it useful for quantum computing,

Anyons become useful for quantum computing when they wind around eachother, so that their world lines form tangles in spacetime. The different tangles represent statesin Hilbertspace, the vast arena of quantum mechanics, and can be used to store information. Hilbertspace is big enough to accommodate very complicated tangles, sohugestorage and bandwidthcould be in the offing.

The theory of anyons has spawned some beautiful but specialized and difficult mathematics, hitherto pursued only by a small cult. I suspect that many of my colleagues have, like me, been waiting for a pretext to dive backin. With these two papers, we haveit. 